\title{
A Comparison Study of the Efficacy of Rapid Titration Quetiapine and Haloperidol in Agitated Adults in an Emergency Setting*
}

\author{
Akikur Mohammad1, Eugene Laska², Marius Campaneau1, Fauzia Syed1', Rebecca Ninah1, \\ Joseph Wanderling', George Simpson" ${ }^{\#}$ \\ ${ }^{1}$ Department of Psychiatry, LAC-USC, Los Angeles, CA, USA \\ ${ }^{2}$ Nathan Kline Institute, New York, NY, USA \\ Email: "gsimpson@usc.edu
}

Received 19 March 2014; revised 18 April 2014; accepted 15 May 2014

Copyright (C) 2014 by authors and Scientific Research Publishing Inc.

This work is licensed under the Creative Commons Attribution International License (CC BY). http://creativecommons.org/licenses/by/4.0/

\section{Open Access}

\section{Abstract}

Objective: Intramuscular (IM) Benzodiazepines and/or Haloperidol alone or with benzodiazepines are frequently used to treat agitation. Based on emerging literature regarding Quetiapine used for the control of anxiety we examined Quetiapine as a possible alternative in selected cases. Methods: This study was a single-blind randomized study comparing Quetiapine PO $(300 \mathrm{mg})$ with a combination of Haloperidol (5 mg), Benztropine mesylate $(2 \mathrm{mg}$ ) and lorazepam $(2 \mathrm{mg}$ ) administered IM to treat agitated patients seeking care in a busy psychiatric emergency setting. Male or female patients (18 - 60), deemed by the attending (admitting) psychiatrist to be indicative of agitated and/or aggressive behavior and had a Positive and Negative Syndrome Score-Excited Component (PANSS-EC), as evaluated by the Research Psychiatrist, and total score equal to or greater than 15. Patients deemed competent were randomized into one of the following treatment groups: Quetiapine 300 or Haloperidol $5 \mathrm{mg}$, benztropine mesylate, or lorazepam given by the IM route. Two scales, PANSS-EC and CGI-C were used to assess patients in the trial. The primary outcome measure PANSS-EC at 2 hours after administration of the medication. Results: Sixty-eight patients were included in the study. There were no significant treatment group differences in baseline condition. There was no significant difference between the two conditions. There was, however, a significant within-group decrease from baseline condition. Conclusion: Finding no significant differences suggests that in general the two treatments were equivalent. To sum up, Quetiapine 300 mg as a single dose appeared safe and effective in agitated patients treated in an ER. The results were similar to a comparison group receiving an intra-muscular combination of Haloperidol, Lo-

\footnotetext{
*Previously presented as a poster at The American College of Neuropsychopharmacology 2009.

This was an investigator initiated study which received grant support from Astra Zeneca,USA. The design implementation data analysis and write up involved only the authors.

\#Corresponding author.
}

How to cite this paper: Mohammad, A., Laska, E., Campaneau, M., Syed, F., Ninah, R., Wanderling, J. and Simpson, G. (2014) A Comparison Study of the Efficacy of Rapid Titration Quetiapine and Haloperidol in Agitated Adults in an Emergency Setting. Open Journal of Psychiatry, 4, 189-194. http://dx.doi.org/10.4236/ojpsych.2014.43025 
razepam and Benztropine. This study has significant limitations. The study was single blind and the use of a placebo would have strengthened the design but would be considered unethical. The sample size was relatively small and the group of patients who come to the ER may not be representative of the population of patients who visit across the country. And finally it was a select subgroup who made up the study population and who were probably less severely ill than other subjects who came to our ER.

\section{Keywords}

Quetiapine (Oral), Haloperidol (Parenteral), Agitation, Excitement, Psychoses

\section{Introduction}

The treatment of agitation in the psychiatric emergency room (ER) has changed little in the last decades. While intramuscular (IM) benzodiazepines are often used, haloperidol (frequently combined with a benzodiazepine and/or an anticholinergic) is still the treatment of choice in many places. Studies of liquid preparations represent a possible alternative for some patients, given their faster absorption compared with tablets or capsules similar to rapid dissolve tablets. Even liquids are not always convenient in a busy ER. The ideal would be a range of options, for example, benzodiazepine or antipsychotic and in turn tablet/capsule, liquid, rapid dissolve tablets or IM preparations. A recent study showed that inhaled loxapine may offer a further option to control agitation, particularly, as peak concentrations are obtained comparable to parenteral administration [1]. The availability of the second-generation antipsychotics (IM preparations) has not altered the situation significantly perhaps because of the increased cost of these preparations. Recently one of us (RM) began to use quetiapine in increasing dosages up to $300 \mathrm{mg}$ in selected patients seeking treatment at the USC ER, based on emerging literature regarding quetiapine use for the control of anxiety [2]-[4].

Based on our initial personal experience, we felt that further study was warranted to examine the potential use of this preparation as a possible alternative in selected cases compared with the IM preparation.

\section{Methods}

This study was a single-blind randomized study comparing Quetiapine PO (300 mg) with a combination of haloperidol (5 mg), benztropine mesylate $(2 \mathrm{mg}$ ) and lorazepam $(2 \mathrm{mg})$ administered IM to treat agitated patients seeking care in a busy psychiatric emergency setting. Male or female patients, aged 18 - 60 years, who presented at the ER with a diagnosis of agitation or aggressive behavior whose symptoms could include restless pacing, inability to sleep, physical aggression towards others as well as other symptoms deemed by the treating (admitting) psychiatrist to be indicative of agitated and/or aggressive behavior were referred to the research team. Patients with a presumed diagnosis of schizophrenia, bipolar disorder, substance abuse and psychosis or other mood disorders with psychosis and had a Positive and Negative Syndrome Score-Excited Component (PANSSEC) (excitement, hostility, tension, uncooperative, poor impulse control) total score of equal to or greater than 15, a Clinical global Impression of Change (CGI-C) of 5 or less and to meet DSM-IV criteria for the diagnosis of psychosis NOS (not otherwise specified) were selected for the study. No attempt was made to control for previous episodes or drug use.

Patients diagnosed with the disorders under study are generally considered to be a vulnerable population. To protect this vulnerable population, the capacity of the patient to consent was determined by a clinician who was independent of the research team. Patients deemed competent and in need of medication were referred to the research team who described the study including the different routes of administration and potential side effects of each medication. Patients excluded from the study received routine treatment. Patients were excluded if they were unable to understand the study, unable to give consent, were receiving anticoagulant therapy, had serious medical illness, were female who were pregnant or nursing, or who had an acute withdrawal from any illicit drugs. Eligible patients were randomized into one of the following treatment groups: Quetiapine $300 \mathrm{mg}$ PO or haloperidol $5 \mathrm{mg}$, benztropine mesylate $2 \mathrm{mg}$, lorazepam $2 \mathrm{mg}$ given by the IM route. Randomization was carried out in advance, blocks of four by a coin toss. Sealed envelopes marked on the outside and with Quetiapine 
or Haloperidol on a folded paper and were prepared in advance and stored in blocks of foam in the nurse's station.

As in a previous study conducted at this site [5], two scales, PANSS-EC and CGI-C were used to assess patients in the trial. The primary outcome measure was PANSS-EC at 2 hours after administration of the medication. The secondary outcome measure was a binary indicator of improvement defined as a CGI-C score of "much improved" or "very much improved" at 2 hours and/or a 20\% reduction in PANSS-EC 2 hours following administration of the medication.

The PANSS-EC was measured at baseline, and at 1, 2, 4 and 8 hours after medication. The CGI-C Severity was measured at baseline and the CGI-C Improvement was measured at 1, 2, 4 and 8 hours after medication. Extrapyramidal symptoms (EPS) were also evaluated at these times. Both lying and sitting blood pressure and pulse were measured at 15 and 45 minutes and at 1, 2, 3, 4, 6 and 8 hours and adverse events were documented throughout the 8-hour study.

\section{Statistical Analysis}

The data are described together with the results of the analysis. Tests of differences in proportions utilized a chisquared statistic. We used an analysis of covariance (ANCOVA) with baseline as the covariate to analyze the primary outcome measure, PANSS-EC at hour 2 for those awake. Note that, formally, this is not an intent-totreat (ITT) analysis since not all patients who were randomized are included in the analysis set. However, arguably, this analysis together with the probability of being awake does include all (but a few) randomized subjects and so might be nearly equivalent to an ITT analysis. The mean time course of the four post treatment PANSSEC evaluations was analyzed using a mixed model repeated measures (MMRM) approach under the assumption that the missing data are "missing at random". This analysis is based on the ITT principle. The MMRM model included terms for age, adjusted baseline, hour, treatment and the two interactions: adjusted baseline by hour and hour by treatment. A mixed model was used to analyze CGI-C binary indicators of improvement. The covariates were the same as those used in the PANSS-EC.

\section{Results}

Sixty-eight patients were included in the study. An additional four patients were randomized to haloperidol but were excluded from the analysis because they had neither baseline nor post-treatment data. There were no significant treatment group differences in baseline PANSS-EC, baseline CGI-C, mean age and in the proportion of males. Further characteristics of the sample are shown in Table 1.

At the 2-hour timepoint, 10 of the 30 haloperidol patients and 9 of the 38 quetiapine patients were asleep at the time the PANNS-EC was administered. These proportions were not significantly different based on a chisquared test (See Table 5). The least square means from the ANCOVA were -11.3 for haloperidol and -11.7 for quetiapine and the contrast was not significant. There was, however, a significant within-group decrease from baseline for both treatments.

A chi-squared test of the secondary outcome variable-20\% or more decrease in PANSS-EC at hour 2-also did not yield a significant treatment difference (see Table 2). Nineteen of the 20 haloperidol patients who were awake and all of the quetiapine patients who were awake had a reduction of at least $20 \%$ in PANSS-EC score.

There were 5 cases among the 68 patients for which the PANSS-EC were missing at all 4-times and these patients were excluded in the MMRM analysis of differences in the time course. Treatment and hour by treatment effects were not significant and there were no significant treatment differences at any hour (see Table 3 for least square means and standard errors from this analysis).

A chi-squared test of improvement based on the CGI-C at hour two yielded a marginally significant treatment effect. All haloperidol patients and 79\% of the quetiapine patients were "much" or "very much" improved $(\mathrm{p}=$ 0.0766). A logistic regression on the same measure, using baseline CGI-C severity as a covariate, failed to converge (likely due to improvement of all haloperidol cases (see Table 4). A second analysis based on the more demanding condition for success of "very much improvement" did converge and the treatment difference was not significant.

There were 4 cases among the 68 patients for which the CGI-C were missing at all 4 time points and these patients were excluded from the mixed model analysis. As in the logistic analyses at hour 2, the model for "much" or "very much" improved failed to converge at hour 2. For the model in which a responder was defined as very 
Table 1. Demographic characteristics.

\begin{tabular}{ccc}
\hline Variable & $\begin{array}{c}\text { IM haloperidol Clocktail } \\
\mathrm{N}=34\end{array}$ & $\begin{array}{c}\text { OralQuetiapine } \\
\mathrm{N}=38\end{array}$ \\
\hline Mean Age & 35.8 & 36.1 \\
Number Men/Women & $28 / 6$ & $29 / 9$ \\
Number by Race & & \\
Black & 9 & 17 \\
White & 7 & 9 \\
Hispanic & 15 & 11 \\
Asian & 1 & 1 \\
Unknown & 2 & 0 \\
\hline
\end{tabular}

Table 2. Number with at least 20\% reduction in PANNS-EC for those awake.

\begin{tabular}{cccccc}
\hline Treatment & 20\% reduction & Hour 1 & Hour 2 & Hour 4 & Hour 8 \\
Haldol & Yes & 24 & 19 & 15 & 0 \\
Cocktail & No & 2 & 29 & 0 & 29 \\
Quetiapine & Yes & 25 & 0 & 0 & 15 \\
& No & 11 & 21 & 1 \\
\hline
\end{tabular}

Table 3. Least square means (SE) for change in PANSS-EC for those awake.

\begin{tabular}{ccccc}
\hline Treatment & Hour 1 & Hour 2 & Hour 4 & -12.8 \\
Haldol & -7.7 & -11.0 & $(0.82)$ & -16.1 \\
Cocktail & $(0.76)$ & $(0.82)$ & -13.2 & $(0.74)$ \\
Quetiapine & -0.64 & $(0.69)$ & $(0.70)$ & $(0.63)$ \\
\hline
\end{tabular}

Table 4. Number with CGI improvement for those awake.

\begin{tabular}{|c|c|c|c|c|c|}
\hline Treatment & CGIImprovement & Hour 1 & Hour 2 & Hour 4 & Hour 8 \\
\hline \multirow{3}{*}{$\begin{array}{c}\text { Haldol } \\
\text { Cocktail }\end{array}$} & Very much & 12 & 14 & 13 & 12 \\
\hline & much & 7 & 6 & 2 & 2 \\
\hline & minimal or worse & 8 & 0 & 0 & 1 \\
\hline \multirow[t]{3}{*}{ Quetiapine } & Very much & 7 & 19 & 13 & 19 \\
\hline & much & 12 & 3 & 3 & 0 \\
\hline & minimal or worse & 17 & 6 & 4 & 2 \\
\hline
\end{tabular}

much improved, the treatment and hour by treatment interaction were not significant and there were no significant treatment differences at any hour. However, there was a marginally significant treatment difference at the first hour. The estimated responder rates were 0.357 for haloperidol and 0.132 for quetiapine $(p=0.0778)$ (Figure 1 shows this as percentage of patients who showed improvement). No patient required a second dose of medication during the eight-hour study period.

\section{Adverse Events}

The adverse event profiles of the two treatments were also similar. Sedation was the most prominent side effect reported for both treatments. As seen in Table 5, some patients were already asleep at the one-hour timepoint, the maximal number occurred at four hours and the effect was still present to a significant degree at 8 hours. During the study, there were 11 patients with low diastolic blood pressures (40 - $60 \mathrm{mmHg}$ ) in the haloperidol group and 12 patients with low diastolic blood pressures in the quetiapine group. This included 4 patients with low blood pressures at baseline.

No cases of syncope or hypotensive collapse were noted. Extra-pyramidal side effects were absent in both groups. 


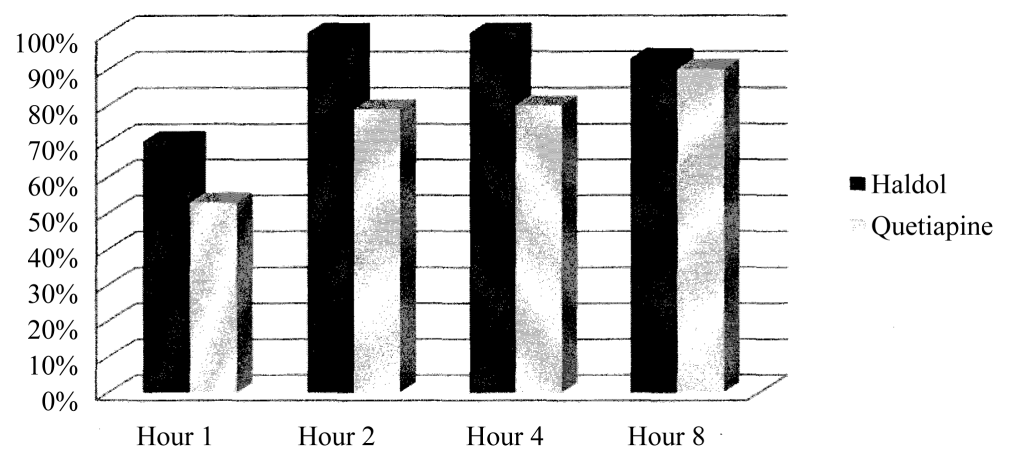

Figure 1. Percentage with CGI improvement much to very much for those awake.

Table 5. Number of patients asleep at the time PANSS-EC and CGI-C* were assessed.

\begin{tabular}{ccccc}
\hline Treatment & Hour 1 & Hour 2 & Hour 4 & Hour 8 \\
\hline Haldol & $4(3)$ & $10(10)$ & $15(15)$ & $14(14)$ \\
Cocktail & $2(2)$ & $9(10)$ & $19(17)$ & $12(13)$ \\
Quetiapine & &
\end{tabular}

*the numbers asleep for CGI-C are in parentheses

\section{Discussion}

Although the finding of no significant differences does not prove that there are no differences, the present study suggests that in general the two treatments were similarly effective in the treatment of acute symptoms of agitation in the emergency setting The haloperidol mixture did have an nearly statistically significant advantage on one of the secondary outcome measures: a binary indicator of improvement defined as a CGI-C score of "much improved" or "very much improved" at 2 hours and/or a 20\% or greater reduction in PANNS-EC 2 hours following administration of the medication. This difference is unlikely to be attributed solely to the patient being asleep, since the treatments did not differ on that measure. However, as might be expected from the more rapid absorption of an I.M injection, changes early on favored the I.M cocktail. It must be noted that no adjustments were made to control the fairly wide error rate to account for the many statistical tests that were conducted.

The use of mixtures of medication (i.e. benzodiazepine plus haloperidol) is based on the belief that the sedative effect of lorazepam is helpful for controlling agitation (short term) [6] [7] and the antipsychotic adds to this and has a longer-term effect. The use of benzodiazepine in the haloperidol "cocktail” was also motivated in part to reduce the amount of haloperidol to an antipsychotic dose and thereby reduce the potential for acute dystonia, akathisia and the serious side effects mentioned below. The rational use of a combination is based on the requirement that it is superior to each of its components used alone and indeed some studies of the haloperidol cocktail do suggest that this is the case [8] [9]. Fortunately, the use of rapid neuroleptization-high doses of high potency antipsychotics is now a thing of the past. This practice was associated with significant side effects, including sudden death, neuroleptic malignant syndrome as well as EPS. It is our impression that in a very busy overcrowded ER, there still seems to be a sense of security around the use of an IM preparation.

To sum up, quetiapine $300 \mathrm{mg}$ as a single dose appeared safe and effective in agitated patients treated in an ER. The results were similar to a comparison group receiving an intra-muscular combination of haloperidol, lorazepam and benztropine. There are suggestions that the IM group responded more rapidly and were more rapidly sedated. The data do reinforce the availability of a range of treatment options for treatment of agitation; liquids, tablets/capsules or even inhalations [6], which in selected patients may avoid the use of intramuscular preparations. This study has significant limitations. The study was single blind, the sample size was relatively small and the select group of patients who come to the ER may not be representative of the population of patients who visit ERs across the country. The study did not include placebo whose use in this clinical context is unethical. No adjustments were made to control the family wise error rate to account for the many statistical tests that were conducted. To demonstrate the equivalence of two treatments based on a possibly more appropriate non-inferiority analysis, requires a substantially larger number of patients than were used here. Finally, since patients who were dozing or appeared asleep were not awakened, it is possible that the number of side effects is 
underestimated and it is probable that the selected subgroup of patients were less severely ill than other patients who are brought to our ER.

\section{Acknowledgements}

Dr. Abderahmane Richane

Dr. Arastou Aminzadeh

Dr. Brenda Quon

Dr. Carolina Bradshaw

Dr. Catherine Scott

Dr. Darin Signorelli

Dr. Gayane Begoyne

Dr. Grace Akra

Dr. Jairo Gomez

Dr. Joseph Mirkovich

Dr. Kamal Bijanpour

Dr. Linda Wang

Dr. Robert Barker

Dr. Simargit Gill

\section{References}

[1] Fishman, R., Casella, J. and Spyker, D. (2009) Inhaled Loxapine Rapidly Improves Acute Agitation in Schizophrenic Patients. Presentation of 162nd Annual Meeting of the American Psychiatric Association, San Francisco, May 2009.

[2] Cutler, A.J. (2001) Quetiapine Effective in Reducing Anxiety: Clinical Evidence. Presented at the American Psychiatric Association Annual Meeting, New Orleans, May 2001.

[3] McFadden, W., et al. (2004) Anti Anxiety Effects of Quetiapine in Bipolar Depression. Presented at the APA 56th Institute on Psychiatric Services, Atlanta, October 2004.

[4] Ganesan, S., et al. (2003) Effectiveness of Quetiapine Treatment of Aggressive Psychosis in the Emergency Psychiatric Setting. Presentation of 156th Annual Meeting of the American Psychiatric Association, San Francisco, May 2003.

[5] Currier, G. and Simpson, G. (2001) Risperidone Liquid Concentrate and Oral Lorazepam versus IM Haloperidol and IM Lorazepam for Treatment of Psychotic Agitation. Journal of Clinical Psychiatry, 62, 153-157. http://dx.doi.org/10.4088/JCP.v62n0303

[6] Campbell, R. and Simpson, G.M. (1986) Alternative Approaches in the Treatment of Psychotic Agitation. Psychosomatics, 27, 23-27. http://dx.doi.org/10.1016/S0033-3182(86)72735-7

[7] Salzman, C., Solomon, D., Miyawaki, E., et al. (1991) Parental Lorazepam versus Parental Haloperidol for the Control of Psychotic Disruptive Behavior. Journal of Clinical Psychiatry.

[8] Battaglia, J., Moss, S., Rush, J., et al. (1997) Haloperidol, Lorazepam, or Both for Psychotic Agitation? A Multicenter, Prospective, Double Blind Emergency Department Study. American Journal of Emergency Medicine, 15, 335-340. http://dx.doi.org/10.1016/S0735-6757(97)90119-4

[9] Bieniek, S.A., Ownby, R.L., Penalver, A., et al. (1998) A Double-Blind Study of Lorazepam versus the Combination of Haloperidol and Lorazepam in Managing Agitation. Pharmacotherapy, 18, 57-62. 
Scientific Research Publishing (SCIRP) is one of the largest Open Access journal publishers. It is currently publishing more than 200 open access, online, peer-reviewed journals covering a wide range of academic disciplines. SCIRP serves the worldwide academic communities and contributes to the progress and application of science with its publication.

Other selected journals from SCIRP are listed as below. Submit your manuscript to us via either submit@scirp.org or Online Submission Portal.
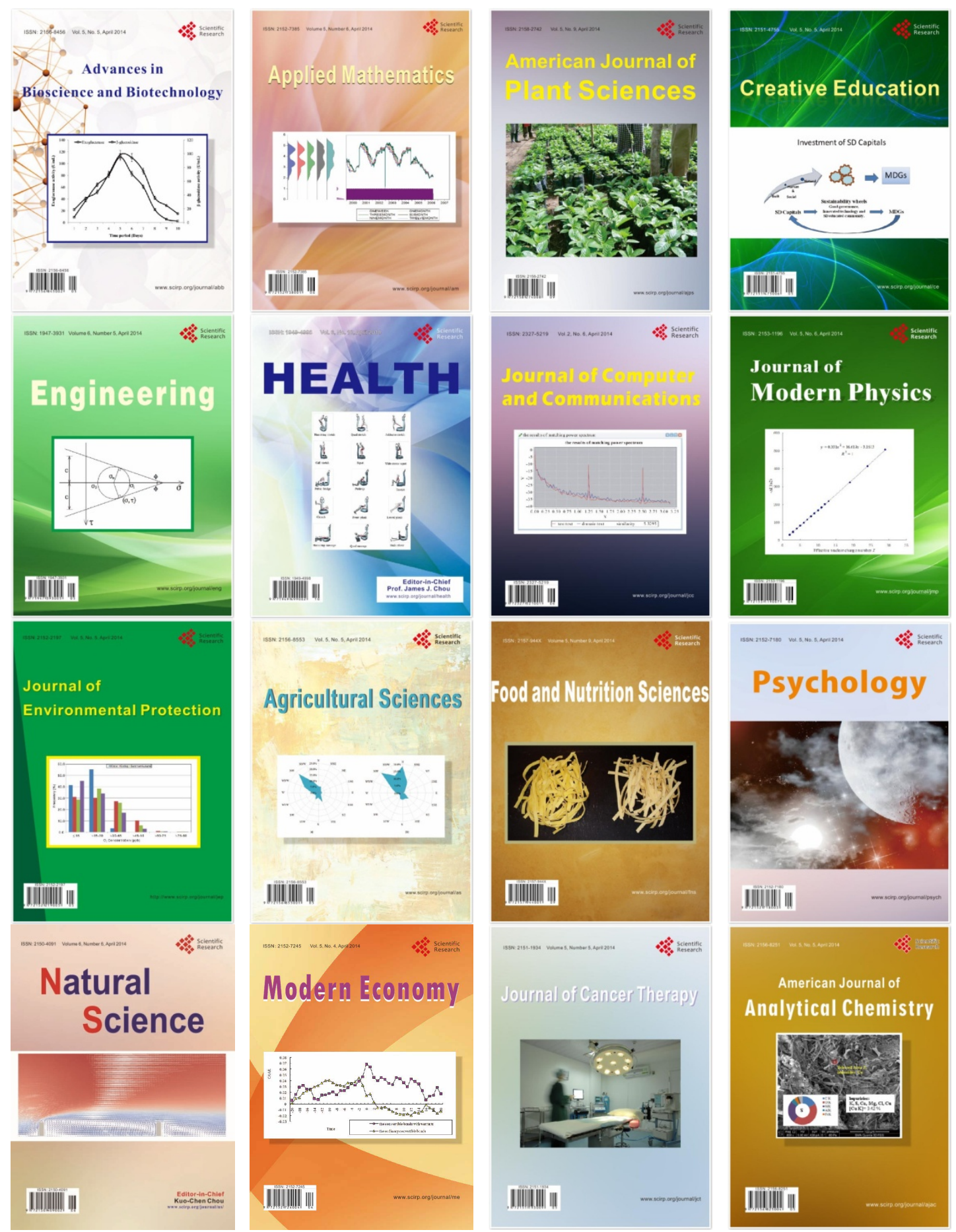